Quim. Nova, Vol. 34, No. 8, 1378-1384, 2011

\title{
SORÇÃO DE ATRAZINA E DE MESOTRIONA EM LATOSSOLOS E ESTIMATIVA DO POTENCIAL DE CONTAMINAÇÃO
}

\author{
Rosane Martinazzo* \\ Departamento de Solos, Faculdade de Agronomia, Universidade Federal do Rio Grande do Sul, Av. Bento Gonçalves, 7712 , \\ 91501-970 Porto Alegre - RS, Brasil
}

Deborah Pinheiro Dick, Mauro Mozael Hirsch e Simone Benvenuti Leite

Departamento de Físico-Química, Instituto de Química, Universidade Federal do Rio Grande do Sul, Av. Bento Gonçalves, 9500 , 91501-970 Porto Alegre - RS, Brasil

Maria do Carmo Ruaro Peralba

Departamento de Química Inorgânica, Instituto de Química, Universidade Federal do Rio Grande do Sul, Av. Bento Gonçalves, 9500, 91501-970 Porto Alegre - RS, Brasil

Recebido em 2/12/10; aceito em 6/4/11; publicado na web em 10/6/11

\begin{abstract}
SORPTION OF ATRAZINE AND MESOTRIONE IN OXISOLS AND ESTIMATION OF CONTAMINATION POTENTIAL. Following application herbicides usually reach the soil and undergo several dissipation processes which determine their effects on target organisms and on the environment. This work aimed to evaluate atrazine and mesotrione sorption in Oxisols and estimate their potential impact on the environment. We observed that atrazine sorption was influenced by soil organic matter content and its hydrophobic character. Clay fraction was the main factor influencing mesotrione sorption followed in a lesser extent by soil organic matter content. In contrast to atrazine, mesotrione retention was favored by the higher carboxylic substitution in the aliphatic chains of soil organic matter. Atrazine and mesotrione were considered compounds that are likely to pose an appreciable risk of causing deleterious effects on the environment.
\end{abstract}

Keywords: isotherm; environmental risk; pesticides.

\section{INTRODUÇÃO}

O manejo integrado de práticas culturais assume fundamental importância na agricultura moderna que busca, simultaneamente, obter elevada produtividade e conservar os recursos naturais. No entanto, muitas vezes o aumento da produtividade ocorre em detrimento da preservação ambiental, principalmente devido ao manejo inadequado do solo e à massiva utilização de agroquímicos. Inúmeros são os casos de contaminação de solo, águas superficiais e subterrâneas e danos à saúde animal e humana, por pesticidas em diversos países, inclusive no Brasil. ${ }^{1-3}$ Em função disso, evidencia-se, cada vez mais, a necessidade de entendimento da dinâmica dos pesticidas no ambiente e de sua avaliação de risco nas condições específicas do local de aplicação.

Diversos modelos matemáticos e/ou critérios de avaliação têm sido desenvolvidos para prever o comportamento dos pesticidas no ambiente e estimar o seu potencial contaminante. Entre os mais utilizados citam-se o método screening da Agência de Proteção Ambiental dos Estados Unidos (USEPA), o índice de vulnerabilidade de águas subterrâneas (GUS), o método de Goss e o índice de impacto ambiental (ERI). Os critérios de screening da USEPA para análise preliminar de riscos de contaminação de águas subterrâneas por pesticidas consideram características como a solubilidade do composto em água $\left(\mathrm{S}_{\mathrm{w}}\right)$, a constante da lei de Henry $\left(\mathrm{K}_{\mathrm{H}}\right)$, o coeficiente de sorção à matéria orgânica do solo $\left(\mathrm{K}_{\mathrm{oc}}\right)$ e o tempo de meia-vida da molécula $\left(\mathrm{t}_{1 / 2}\right)$ no solo e na água. ${ }^{4} \mathrm{O}$ índice de vulnerabilidade de águas subterrâneas (Groundwater Ubiquity Score - GUS), proposto por Gustafson, ${ }^{5}$ utiliza os valores de $\mathrm{t}_{1 / 2}$ do herbicida no solo e o $\mathrm{K}_{\mathrm{oc}}$

*e-mail: martinazzo_ro@yahoo.com.br como critérios de classificação do seu potencial de lixiviação. O risco de contaminação das águas superficiais, de acordo com os critérios propostos por Goss, ${ }^{6}$ considera o potencial de transporte do pesticida associado ao sedimento e dissolvido na água e utiliza as variáveis $\mathrm{S}_{\mathrm{w}}$, $\mathrm{t}_{1 / 2}$ e $\mathrm{K}_{\mathrm{oc}}$ do composto. O índice de risco ambiental (Environmental Risk Index-ERI), proposto por Alister e Kogan, ${ }^{7}$ considera a toxicologia, o potencial de lixiviação e de volatilização e a persistência do pesticida.

De modo geral, esses métodos de avaliação possuem grande utilidade devido à simplicidade de uso e por substituírem, de certa forma, estudos complexos e de elevados custos realizados em campo. Adicionalmente, os critérios para estimativa do potencial de risco podem ser utilizados em avaliações preliminares para selecionar os compostos a serem avaliados em estudos mais detalhados, conforme realizado por Primel et al.. ${ }^{8}$ Contudo, a limitação desses métodos reside na falta de informações precisas sobre os parâmetros de entrada utilizados por eles para condições específicas de solo e clima do local de interesse. Segundo Pessoa e Scramin, ${ }^{9}$ a utilização de dados médios disponibilizados na literatura científica pode induzir a erros de interpretações de tendências futuras. Os autores citam que esse fato é constatado principalmente para duas das principais entradas de dados, solicitadas por quase todos os modelos e critérios de análise do comportamento de agrotóxicos em solos: o $\mathrm{K}_{\mathrm{oc}}$ e o $\mathrm{t}_{1 / 2}$ do produto. Esses parâmetros possuem grande amplitude de variação, por serem influenciados pelas condições climáticas e características do solo da região avaliada. ${ }^{9}$

Em função disso, este estudo se propôs a avaliar o comportamento sortivo de dois herbicidas em amostras de horizonte A de seis latossolos do Estado do Rio Grande do Sul e estimar o potencial contaminante desses compostos pelo uso do método screening da USEPA, do 
índice GUS, do método de Goss e do ERI. Os herbicidas avaliados foram a atrazina [2-cloro-4-etilamino-6-isopropilamino-s-triazina] e a mesotriona [2-(4-mesil-2-nitrobenzoil) ciclo-hexano-1,3-diona], os quais representam grupos de compostos com diferentes mecanismos de ação e propriedades físico-químicas contrastantes. Adicionalmente, a atrazina é considerada um dos produtos mais utilizados no Brasil para o controle de plantas invasoras de folhas largas, ${ }^{10}$ enquanto que a mesotriona é um produto relativamente novo e tem sido recomendada em substituição à atrazina ou em associação com a mesma nas plantações de milho, ${ }^{11}$ a fim de reduzir as doses de aplicação e, consequentemente, o impacto ambiental de ambos.

\section{PARTE EXPERIMENTAL}

\section{Local de coleta e características dos solos avaliados}

Amostras de solo foram coletadas em triplicata no horizonte A de perfis modais de 6 latossolos sob vegetação nativa, no Estado do Rio Grande do Sul. As principais características dos solos e dos locais de coleta das amostras são apresentadas na Tabela 1. Os teores de carbono (C) e de nitrogênio (N) das amostras de solo foram determinados em analisador elementar (Perkin Elmer 2400) e as análises de K, Ca, Mg e $\mathrm{Al}$ trocáveis e de $\mathrm{pH}$ seguiram metodologia descrita por Tedesco et al. ${ }^{12}$ (Tabela 2). As análises foram realizadas em amostras de solo compostas, secas ao ar e peneiradas em malha de $2 \mathrm{~mm}$. A composição química da matéria orgânica dos respectivos solos foi previamente estudada por Dick et al. ${ }^{13}$ e Dalmolin et al.. ${ }^{14}$

\section{Ensaios de sorção dos herbicidas}

Os dois herbicidas empregados neste estudo (Figura 1S, material suplementar) se diferenciam quanto às suas propriedades físicoquímicas. A atrazina é um herbicida de caráter básico com $\mathrm{pK}_{\mathrm{a}} 1,7$, solubilidade em água $33 \mathrm{mg} \mathrm{L}^{-1}, \log \mathrm{K}_{\mathrm{ow}} 2,34, \mathrm{~K}_{\mathrm{H}} 1,5 \times 10^{-4} \mathrm{~Pa} \mathrm{~m}^{3}$ $\mathrm{mol}^{-1}$ e pressão de vapor $2,89 \times 10^{-7} \mathrm{~mm} \mathrm{Hg}$. A mesotriona comportase como ácido fraco apresentando $\mathrm{pK}_{\mathrm{a}} 3,1$, solubilidade em água 160 $m g \mathrm{~L}^{-1}, \log \mathrm{K}_{\mathrm{ow}} 0,11, \mathrm{~K}_{\mathrm{H}} 5,1 \times 10^{-7} \mathrm{~Pa} \mathrm{~m}^{3} \mathrm{~mol}^{-1}$ e pressão de vapor $4,27 \times 10^{-8} \mathrm{~mm} \mathrm{Hg} .^{7,15}$ Os compostos atrazina e mesotriona em fase sólida foram obtidos por secagem dos produtos comerciais Gesaprim GRDA (880 g L ${ }^{-1}$, Syngenta) e Callisto (480 g L ${ }^{-1}$, Syngenta), respectivamente, conforme metodologia descrita por Ávila et al.. ${ }^{16}$ As concentrações finais de atrazina e de mesotriona nas amostras secas foram 86,2 e $85 \%$, respectivamente, as quais foram obtidas por análise elementar (Perkin Elemer 2400) e calculadas em função da proporção ponderal teórica de nitrogênio presente nos compostos. ${ }^{16}$
As soluções padrão primário $\left(30 \mathrm{mg} \mathrm{L}^{-1}\right)$ de atrazina e de mesotriona foram preparadas em meio de $\mathrm{CaCl}_{2} 0,01 \mathrm{~mol} \mathrm{~L}^{-1} \mathrm{e}$ agitadas durante $24 \mathrm{~h}$ (160 rpm, GFL 1092), no escuro, a $20 \pm 2{ }^{\circ} \mathrm{C}$, para garantir a completa solubilização dos compostos. Soluções secundárias nas concentrações de 0 a $30 \mathrm{mg}$ de atrazina $\mathrm{L}^{-1}$ e de 0 a $50 \mathrm{mg}$ de mesotriona $\mathrm{L}^{-1}$ foram preparadas e utilizadas para a construção das curvas de calibração e das isotermas de sorção. Para a obtenção das isotermas $0,5 \mathrm{~g}$ de solo e $25 \mathrm{~mL}$ de solução foram adicionados em tubo de centrífuga ( $50 \mathrm{~mL}$, polietileno) em sistema hermeticamente fechado. Cada ponto da isoterma foi preparado em duplicata. Após $24 \mathrm{~h}$ de agitação (160 rpm, GFL 1092), em ambiente protegido da luz e temperatura de $20 \pm 2{ }^{\circ} \mathrm{C}$, as soluções foram centrifugadas (1529 $g, 10 \mathrm{~min}$ ) e filtradas. $\mathrm{O}$ volume dos sobrenadantes foi medido e os mesmos foram armazenados em frascos de vidro âmbar a $4 \pm 2{ }^{\circ} \mathrm{C}$ para posterior filtração em filtro de celulose $(0,45 \mu \mathrm{m}$, Sartorious Biolab) no caso da mesotriona, e extração em fase sólida (SPE) e filtração em sulfato de sódio e lã de vidro no caso da atrazina. $\mathrm{O}$ pH foi monitorado na solução sobrenadante do teste em branco (solo + $\mathrm{CaCl}_{2}$ 0,01 mol L-1) e das amostras logo após a centrifugação. Os valores obtidos situaram-se em torno de 5,5 $\pm 0,5$.

A quantificação da atrazina foi feita por cromatografia gasosa (GC), de acordo com a metodologia de Kleinschmitt, ${ }^{17}$ utilizando-se um cromatógrafo GC 17 A (Shimadzu), equipado com injetor split/ splitless, detector de ionização de chama (FID) e coluna capilar SPB-5 [poli (5\% difenil - 95\% dimetilsiloxano)] de $30 \mathrm{~m}$ x 0,25 mm x $0,25 \mu \mathrm{m}$. Utilizou-se rampa de aquecimento iniciando em $130{ }^{\circ} \mathrm{C}$ durante $1 \mathrm{~min}$, seguida de um aumento de $10^{\circ} \mathrm{C} \mathrm{min}^{-1}$ até $190{ }^{\circ} \mathrm{C}$, após $15^{\circ} \mathrm{C} \mathrm{min}{ }^{-1}$ até $280^{\circ} \mathrm{C}$ e, por fim, temperatura constante de 280 ${ }^{\circ} \mathrm{C}$ durante $1 \mathrm{~min}$. A temperatura do injetor e do detector foi $250 \mathrm{e}$ $295^{\circ} \mathrm{C}$, respectivamente. O hidrogênio 5.0 (White Martins) foi usado como gás de arraste e o volume de injeção foi de $1 \mu \mathrm{L}$.

A mesotriona foi determinada por cromatografia líquida (HPLC) utilizando-se um método concedido pela empresa produtora do herbicida, com algumas adaptações. Utilizou-se um cromatógrafo LC 10 A (Shimadzu) acoplado a um detector UV/Vis (SPD 10 A, Shimadzu), forno com temperatura ambiente, loop de amostra de 20 $\mu \mathrm{L}$, coluna Supelcosil LC-18 (Supelco) de 4,6 mm x $25 \mathrm{~cm}$ x $5 \mu \mathrm{m}$ e pré-coluna de $4 \mathrm{~mm}$ x $4 \mathrm{~cm}$. A mistura acetonitrila:água (40:60) foi usada como fase móvel em sistema isocrático com fluxo de $1 \mathrm{~mL}$ $\min ^{-1}$. Previamente à análise, a fase móvel foi filtrada em membrana de nylon $(0,45 \mu \mathrm{m}$, Sartorious) e degaseificada com gás hélio (White Martins). Os limites analíticos de detecção e de quantificação do método foram 0,09 e $0,28 \mathrm{mg} \mathrm{L}^{-1}$, respectivamente. ${ }^{18}$

A quantidade de atrazina ou de mesotriona sorvida ao solo foi calculada pela diferença entre a concentração inicial adicionada ao

Tabela 1. Solo, local de amostragem, profundidade do horizonte A amostrado, classificação, mineralogia da fração argila e teores de óxidos de ferro dos latossolos avaliados

\begin{tabular}{|c|c|c|c|c|c|c|}
\hline Solo $^{\mathrm{a}}$ & Local & Prof. (cm) & Classificação & Mineralogia $^{\mathrm{b}}$ & $\mathrm{Fe}_{\mathrm{d}}^{\mathrm{c}}$ & $\mathrm{Fe}_{\mathrm{o}}{ }^{\mathrm{c}}$ \\
\hline LV-AR & André da Rocha & $0-25$ & Latossolo Vermelho distrófico & n.d. & 55,3 & 1,8 \\
\hline LV-CA & Cruz Alta & $0-35$ & Latossolo Vermelho distrófico & $\mathrm{Ct} / \mathrm{Gt} / \mathrm{Hm}$ & 2,0 & 0,8 \\
\hline LV-ER & Erechim & $0-22$ & Latossolo Vermelho aluminoférrico & $\mathrm{Ct} / \mathrm{Gt} / \mathrm{Hm}$ & 101,0 & 4,3 \\
\hline LV-PF & Passo Fundo & $0-33$ & Latossolo Vermelho distrófico & $\mathrm{Ct} / \mathrm{Hm} / \mathrm{Gt}$ & 34,0 & 2,0 \\
\hline LV-SA & Santo Ângelo & $0-23$ & Latossolo Vermelho distroférrico & $\mathrm{Ct} / \mathrm{Hm} / \mathrm{Gt}$ & 101,1 & 2,4 \\
\hline LB-VA A ${ }^{\dagger}$ & Vacaria & $0-30$ & Latossolo Bruno aluminoférrico & $\mathrm{Ct} / \mathrm{Gt} / \mathrm{Hm}$ & 106,0 & 3,1 \\
\hline LB-VA $A_{2}^{\ddagger}$ & Vacaria & $30-60$ & Latossolo Bruno aluminoférrico & $\mathrm{Ct} / \mathrm{Gt} / \mathrm{Hm}$ & 100,0 & 2,1 \\
\hline
\end{tabular}

aLatossolo Vermelho dos municípios de André da Rocha (LV-AR), Cruz Alta (LV-CA), Erechim (LV-ER), Passo Fundo (LV-PF) e Santo Ângelo (LV-SA) e Latossolo Bruno do município de Vacaria (LB-VA). ${ }^{\dagger}$ Horizonte $\mathrm{A}_{1}$; ${ }^{*}$ horizonte $\mathrm{A}_{2}$. ${ }^{\mathrm{b}} \mathrm{Ct}$ : caulinita, Hm: hematita, Gt: goetita, n.d.: não disponível. Fontes: refs. 13 e 14. ${ }^{C} \mathrm{Fe}_{\mathrm{d}}$ : teor de ferro extraível por ditionito-citrato-bicarbonato e $\mathrm{Fe}_{\mathrm{o}}$ : teor de ferro extraível por oxalato de amônio. Fontes: refs. 13 , 14 e 29 
Tabela 2. Solo, teores de argila, de carbono e de nitrogênio, $\mathrm{pH}$ em água, capacidade de troca catiônica efetiva $\left(\mathrm{CTC}_{\mathrm{ef}}\right)$ e teores de cálcio, magnésio, alumínio e potássio disponível nos latossolos avaliados

\begin{tabular}{|c|c|c|c|c|c|c|c|c|c|}
\hline Solo $^{a}$ & $\begin{array}{l}\text { Argila } \\
\mathrm{g} \mathrm{kg}^{-1}\end{array}$ & $\begin{array}{c}\mathrm{C} \\
\mathrm{g} \mathrm{kg}^{-1}\end{array}$ & $\begin{array}{c}\mathrm{N} \\
\mathrm{g} \mathrm{kg}^{-1}\end{array}$ & $\mathrm{pH}$ & $\begin{array}{c}\mathrm{CTC}_{\mathrm{ef}} \\
\mathrm{cmol}_{\mathrm{c}} \mathrm{dm}^{-3}\end{array}$ & $\begin{array}{c}\mathrm{Ca} \\
\mathrm{cmol}_{\mathrm{c}} \mathrm{dm}^{-3}\end{array}$ & $\begin{array}{c}\mathrm{Mg} \\
\mathrm{cmol}_{\mathrm{c}} \mathrm{dm}^{-3}\end{array}$ & $\begin{array}{c}\mathrm{Al} \\
\mathrm{cmol}_{\mathrm{c}} \mathrm{dm}^{-3}\end{array}$ & $\begin{array}{c}\mathrm{K} \\
\mathrm{cmol}_{\mathrm{c}} \mathrm{dm}^{-3}\end{array}$ \\
\hline LV-AR & 560,0 & 38,3 & 2,9 & 4,8 & 6,4 & 1,0 & 0,9 & 4,1 & 0,18 \\
\hline LV-CA & 203,0 & 9,5 & 1,1 & 4,7 & 4,1 & 1,6 & 0,6 & 1,8 & 0,07 \\
\hline LV-ER & 522,0 & 31,9 & 2,4 & 4,9 & 8,2 & 3,5 & 2,8 & 1,7 & 0,24 \\
\hline LV-PF & 367,0 & 27,5 & 1,3 & 4,4 & 6,1 & 1,0 & 0,8 & 4,2 & 0,12 \\
\hline LV-SA & 625,0 & 21,5 & 1,3 & 4,8 & 5,0 & 1,6 & 1,1 & 2,2 & 0,13 \\
\hline LB-VA $A_{1}^{\dagger}$ & 524,0 & 37,5 & 3,2 & 4,1 & 9,1 & 3,1 & 2,4 & 3,4 & 0,19 \\
\hline LB-VA A ${ }_{2}^{\ddagger}$ & 650,0 & 26,7 & 1,8 & 4,7 & 6,7 & 0,9 & 0,6 & 5,1 & 0,08 \\
\hline
\end{tabular}

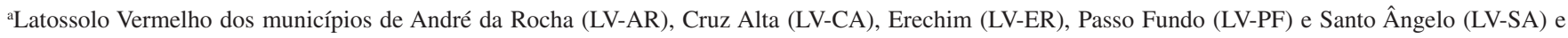
Latossolo Bruno do município de Vacaria (LB-VA). ${ }^{\dagger}$ Horizonte $\mathrm{A}_{1}$; ; thorizonte $\mathrm{A}_{2}$.

solo (Ci) e a concentração em equilíbrio após a sorção (Ce). Nesse cálculo, a área do pico do teste em branco $\left(\mathrm{Ce}_{\text {branco }}\right)$ foi subtraída da área do pico das amostras $\left(\mathrm{Ce}_{\text {amostra }}\right)$ para excluir possíveis interferências de compostos coextraídos. A partir da equação linear da isoterma de sorção obteve-se o coeficiente de sorção ao solo $-K_{d}$, e o coeficiente de sorção à fração orgânica - $\mathrm{K}_{\mathrm{oc}}$, que normaliza o $\mathrm{K}_{\mathrm{d}}$ para o teor de carbono total do solo.

Aos dados de sorção foram aplicados os modelos de Boltzmann, Freundlich e linear. A qualidade dos ajustes dos modelos aos dados e a sua adequabilidade foram analisadas por meio do coeficiente de correlação entre os valores observados e os valores preditos pelos modelos (r), da probabilidade de erro (p) e do gráfico de dispersão de resíduos padronizados (GRES) proposto por Souza et al..$^{19}$ Adicionalmente, foram feitas análises de correlação entre o coeficiente de sorção e os atributos do solo e da matéria orgânica, utilizando-se o pacote estatístico Origin 7.0 SR0 (OriginLab Corporation, 2002).

\section{Avaliação do potencial de contaminação}

O risco potencial de contaminação dos dois herbicidas avaliados foi investigado pelos métodos: screening da USEPA e índice GUS, para caracterizar os compostos segundo o risco de contaminação de águas subterrâneas; Goss, para avaliação do potencial de contaminação de águas superficiais e, ERI, índice de risco ambiental.

O método de screening da USEPA considera os seguintes critérios para caracterizar os pesticidas de alto potencial contaminante: solubilidade em água $>30 \mathrm{mg} \mathrm{L}^{-1} ; \mathrm{K}_{\mathrm{oc}}<300-500 \mathrm{~mL} \mathrm{~g}^{-1}$; constante da lei de Henry $\left(\mathrm{K}_{\mathrm{H}}\right)<10^{-2} \mathrm{~Pa} \mathrm{~m}^{3} \mathrm{~mol}^{-1}$; especiação (Esp) - negativamente carregado na faixa de $\mathrm{pH}$ do solo (5 a 8); $\mathrm{t}_{1 / 2}$ no solo $>14-21$ dias e, $\mathrm{t}_{1 / 2}$ na água $>175$ dias. ${ }^{4}$ Caso não atendam a esses critérios, os compostos são considerados de potencial intermediário de contaminação ou não contaminantes.

O índice GUS (Equação 1) estabelece que compostos com valores $<1,8$ são não lixiviáveis e aqueles com GUS $>2,8$ são lixiviáveis. Pesticidas com valores entre 1,8 e 2,8 são considerados moderadamente lixiviáveis. ${ }^{5} \mathrm{O} \mathrm{t}_{1 / 2}$ da atrazina no solo considerado neste trabalho foi de 45,9 dias, referente à média dos valores obtidos para solos brasileiros sob vegetação nativa avaliados em estudos anteriores $^{20} \mathrm{e} \mathrm{o} \mathrm{t}_{1 / 2}$ na água foi de 86 dias, conforme reportado no Pesticide Properties Database. ${ }^{15}$ No caso da mesotriona, devido à escassez de dados de persistência em solos brasileiros, optou-se pelo uso do maior $t_{1 / 2}$ (34 dias) reportado em estudos com 17 solos da Europa e dos EUA, ${ }^{21,22}$ para simular uma situação extrema de impacto da mesotriona no ambiente. Nesses estudos os autores obtiveram valores de $t_{1 / 2}$ variando entre 4,5 e 34 dias. Em água, a mesotriona é considerada estável. ${ }^{15}$

$$
\text { GUS }=\log \mathrm{t}_{1 / 2}\left(4-\log \mathrm{K}_{\mathrm{oc}}\right)
$$

Os critérios considerados para a classificação dos pesticidas quanto ao risco de contaminação de águas superficiais podem ser encontrados em Goss. ${ }^{6} \mathrm{O}$ método considera o potencial de transporte de resíduos associados ao sedimento e/ou dissolvidos em água, sendo os compostos classificados como de alto, médio ou baixo potencial de contaminação.

O ERI (Equação 2) considera cinco fatores de impacto: a persistência do pesticida no solo $(\mathrm{P})$, caracterizada pelo $\mathrm{t}_{1 / 2}$, o potencial de lixiviação (L), obtido pelo índice de lixiviação LIX, a volatilidade (V), o perfil toxicológico do composto (TP) e a dose de aplicação em campo (D). ${ }^{7}$

$$
\mathrm{ERI}=(\mathrm{P}+\mathrm{L}+\mathrm{V}+\mathrm{TP}) * \mathrm{D}
$$

Cada termo da equação acima possui quatro graus de severidade (baixo, médio, alto e muito alto) aos quais são atribuídos valores de 1 a 4, respectivamente, utilizados no cálculo do ERI. Os intervalos de valores propostos para cada grau de severidade encontram-se detalhadamente descritos em Alister e Kogan. ${ }^{7}$ Para obtenção do fator persistência, considerou-se $t_{1 / 2}$ como sendo 45,9 e 34 dias, respectivamente para a atrazina e a mesotriona, conforme descrito anteriormente.

O índice LIX foi calculado segundo a Equação 3, proposta por Spadotto, ${ }^{23}$ considerando a taxa de degradação do composto $(\mathrm{k})$ e o $\mathrm{K}_{\mathrm{oc}}$. $\mathrm{O}$ índice varia de 0 a 1 , sendo que pesticidas com índice LIX $=0$ são considerados não lixiviáveis e aqueles com LIX $\geq 0,1$ são ditos lixiviáveis. Os compostos cujo índice se encontra entre 0 e 0,1 possuem potencial intermediário de lixiviação. Os valores de $\mathrm{k}$ considerados neste estudo foram 0,013 e $0,069 \mathrm{dia}^{-1}$ para a atrazina e a mesotriona, respectivamente.

$$
\mathrm{LIX}=\exp \left(-\mathrm{k} * \mathrm{~K}_{\mathrm{oc}}\right)
$$

O potencial de volatilização foi obtido pela Equação 4, a qual se refere a um cálculo simplificado por Alister e Kogan, ${ }^{7}$ onde a perda de um pesticida no campo é expressa como uma função da pressão de vapor (PV) e do peso molecular do composto (M).

$$
\mathrm{V}=2,9 \times 10^{-3} * \mathrm{PV} * \mathrm{M}^{0,5}
$$

O perfil toxicológico (Equação 5) considera quatro fatores que caracterizam a toxicologia de um composto e estima o impacto que o mesmo teria caso atingisse o compartimento biótico. Para estimar o TP foram considerados o coeficiente de partição octanol-água 
$\left(\mathrm{K}_{\mathrm{ow}}\right)$, a dose de referência ( $\left.\mathrm{Rfd}\right)$, a dose letal $\left(\mathrm{LD}_{50}\right)$ e a toxicologia animal (TA).

$$
\mathrm{TP}=\mathrm{K}_{\mathrm{ow}}+\mathrm{Rfd}+\mathrm{LD}_{50}+\mathrm{TA}
$$

$\mathrm{OK}_{\mathrm{ow}}$ representa uma estimativa do potencial de bioconcentração de um dado pesticida em plantas e animais. Valores de $\log \mathrm{K}_{\mathrm{ow}}$ acima de 3,0 indicam que o composto possui um elevado potencial de acumulação em ácidos graxos. ${ }^{7}$ A Rfd é uma estimativa da exposição diária aceitável a um dado pesticida por uma pessoa sem que isso cause riscos à sua saúde. A Rfd é extrapolada para humanos a partir de estudos experimentais com animais e é considerada um indicador do risco que um pesticida representaria para uma dada população humana caso fosse sujeita à exposição crônica do pesticida. Valores de Rfd menores que $0,1 \mathrm{mg} \mathrm{kg}^{-1}$ dia $^{-1}$ são considerados de baixo risco. ${ }^{7}$ $\mathrm{A} \mathrm{LD}_{50}$ corresponde à concentração do pesticida capaz de matar $50 \%$

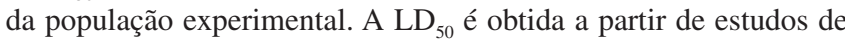
dose-resposta feitos com animais e é considerada um indicativo da toxicologia aguda do pesticida para humanos. $\mathrm{O}$ fator TA representa o impacto que um dado pesticida teria sobre a fauna caso atingisse um local não alvo, como uma lagoa ou um rio. Esse fator considera a $\mathrm{LD}_{50}$ para insetos, pássaros e organismos aquáticos. ${ }^{7}$

No presente estudo foram considerados valores reportados anteriormente por Alister e Kogan, ${ }^{7}$ sendo para a atrazina: $\log \mathrm{K}_{\mathrm{ow}}=$ 2,34, $\mathrm{Rfd}=0,035 \mathrm{mg} \mathrm{kg}^{-1} \mathrm{dia}^{-1}, \mathrm{LD}_{50}$ para humanos $=3000 \mathrm{mg} \mathrm{kg}^{-1}$, $\mathrm{LD}_{50}$ para patos $=10000 \mathrm{mg} \mathrm{kg}-1, \mathrm{LD}_{50}$ para truta $=9,9 \mathrm{mg} \mathrm{kg}^{-1} \mathrm{e}$ $\mathrm{LD}_{50}$ para abelha $=100 \mathrm{mg} \mathrm{kg}^{-1}$, e para a mesotriona: $\log \mathrm{K}_{\mathrm{ow}}=0,11$, $\mathrm{Rfd}=0,02 \mathrm{mg} \mathrm{kg}^{-1} \mathrm{dia}^{-1}, \mathrm{LD}_{50}$ para humanos $=2000 \mathrm{mg} \mathrm{kg}^{-1}, \mathrm{LD}_{50}$ para patos $=5200 \mathrm{mg} \mathrm{kg}^{-1}, \mathrm{LD}_{50}$ para truta $=120 \mathrm{mg} \mathrm{kg}^{-1} \mathrm{e} \mathrm{LD}_{50}$ para abelha $=100 \mathrm{mg} \mathrm{kg}^{-1}$. Somados os valores de referência obteve-se
$\mathrm{TP}=13$ para a atrazina e $\mathrm{TP}=8$ para a mesotriona, os quais foram incluídos na Equação 2 para o cálculo do ERI.

O último fator a ser considerado no cálculo do ERI é a dose de aplicação e, no presente estudo, foi considerada como sendo $3 \mathrm{~kg}$ i.a. ha $^{-1}$ para a atrazina e $0,192 \mathrm{~kg}$ i.a ha ${ }^{-1}$ para a mesotriona.

\section{RESULTADOS E DISCUSSÃO}

\section{Comportamento sortivo}

Considerando-se apenas os parâmetros comumente empregados para comparação estatística, como o coeficiente de correlação e a probabilidade de erro $(<5 \%)$, os modelos linear e de Boltzmann se apresentaram como igualmente adequados para descrever os dados de sorção de atrazina (Figura 1) e de mesotriona (Figura 2S, material suplementar) para os solos avaliados. Em função disso, para fins de comparação entre os solos, optou-se pela obtenção do $\mathrm{K}_{\mathrm{d}}$ pelo uso do modelo linear. O modelo de Freundlich ajustou-se apenas aos dados de sorção de atrazina nos solos LV-AR e LB-VA $\mathrm{A}_{1}$ e de mesotriona nos solos LV-AR, LV-ER, LB-VA $A_{1}$ e LB-VA $\mathrm{A}_{2}$. Contudo, de acordo com o GRES, o modelo de Boltzmann foi o que melhor se ajustou aos dados de sorção de atrazina e de mesotriona nos solos avaliados, exceto para a sorção de mesotriona no LV-CA e no LV-SA, nos quais o modelo linear apresentou melhor ajuste (dados não apresentados). Isso indica que para a maioria dos solos avaliados a afinidade do sorbato pelo sorbente aumenta à medida que progride a sorção. A isoterma de sorção de atrazina do LV-CA não foi apresentada devido à baixa sorção do herbicida nesse solo, o que originou um elevado coeficiente de variação e baixa confiabilidade dos dados.
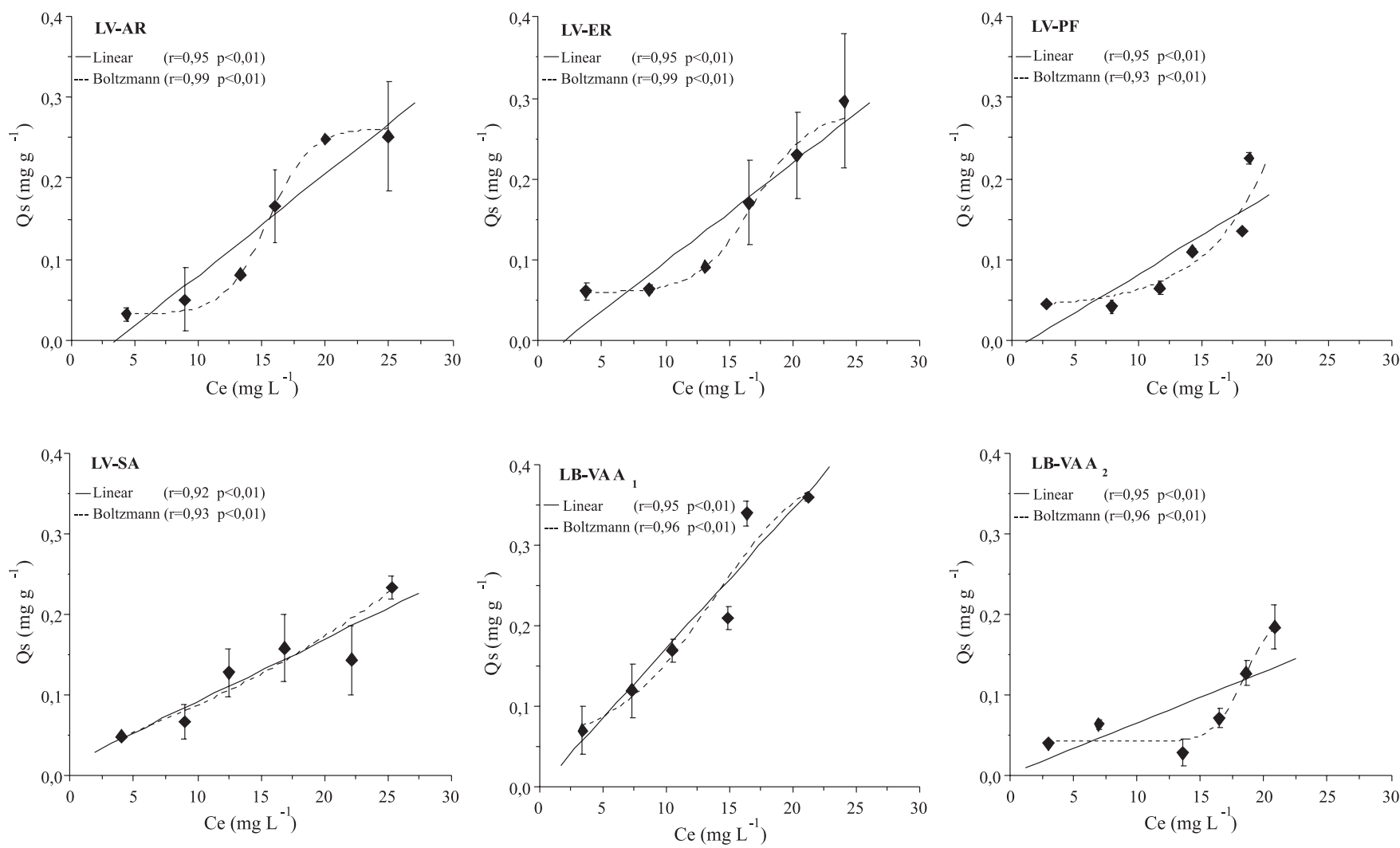

Figura 1. Isotermas de sorção de atrazina em amostras de horizonte A de Latossolo Vermelho dos municípios de André da Rocha (LV-AR), Erechim (LV-ER), Passo Fundo (LV-PF) e Santo Ângelo (LV-SA), e em amostras de horizonte $A_{1}$ e $A_{2}$ de Latossolo Bruno do município de Vacaria (LB-VA). Os símbolos do gráfico representam a média de duas repetições e as barras de erro indicam o desvio padrão da média e não aparecem quando menores que o símbolo para a média 
Os coeficientes de sorção de atrazina nos latossolos avaliados (Tabela 3) encontram-se dentro da faixa de valores reportada para solos brasileiros. ${ }^{17,24,25}$ No entanto, ampla variabilidade nos valores de $\mathrm{K}_{\mathrm{d}}$ pode ser observada nos dados da literatura (Tabela $1 \mathrm{~S}$, material suplementar) devido, provavelmente, às diferenças nas características dos solos e nas condições experimentais utilizadas. No presente estudo, em geral, os maiores valores de $\mathrm{K}_{\mathrm{d}}$ foram observados nos solos que apresentaram maior teor de carbono (Tabela 2), indicando que a fração orgânica é o principal sorbente de atrazina no solo, conforme já reportado anteriormente. ${ }^{24,25} \mathrm{Em}$ estudo realizado com quatro classes de solos do Rio Grande do Sul, Dick et al. ${ }^{25}$ observaram que a matéria orgânica contribuiu com 56 a $85 \%$ da retenção de atrazina no solo. Contudo, não somente o teor de matéria orgânica contribui para a retenção desse herbicida no solo, mas também suas características químicas e estruturais. ${ }^{25,26}$ No presente estudo, das correlações testadas entre o $\mathrm{K}_{\mathrm{d}}$ e os atributos químicos da matéria orgânica dos solos estudados (Tabela 2S, material suplementar), apenas resultou significativa aquela realizada com o índice $\mathrm{C}$-alquil/C-carboxílico $(\mathrm{r}=0,88 ; \mathrm{p}<0,02)$. Esse índice pode ser considerado um indicativo da funcionalização da cadeia alquila, onde menores valores sugerem menor funcionalização. ${ }^{26}$ A correlação obtida indica que com o aumento do tamanho da cadeia alquila a sorção de atrazina pela matéria orgânica é favorecida. Resultados similares foram reportados por Dick et al..$^{25}$ em estudo com Latossolo, Vertissolo, Argissolo e Planossolo. Possivelmente, a menor funcionalização da cadeia alifática favorece a conformação globular da estrutura micelar da matéria orgânica, aumentando assim os sítios hidrofóbicos alifáticos, pelos quais a atrazina apresenta maior afinidade. ${ }^{25}$,

Tabela 3. Coeficientes de sorção de atrazina e de mesotriona ao solo $\left(K_{d}\right)$ e à fração orgânica $\left(\mathrm{K}_{\mathrm{oc}}\right)$ e respectivos desvios padrão nos latossolos avaliados

\begin{tabular}{|c|c|c|c|c|}
\hline \multirow{2}{*}{ Solo $^{\mathrm{a}}$} & \multicolumn{2}{|c|}{$\mathrm{K}_{\mathrm{d}}\left(\mathrm{L} \mathrm{kg}^{-1}\right)$} & \multicolumn{2}{|c|}{$\mathrm{K}_{\mathrm{oc}}\left(\mathrm{L} \mathrm{kg}^{-1}\right)$} \\
\hline & Atrazina & Mesotriona & Atrazina & Mesotriona \\
\hline LV-AR & $12,5 \pm 1,5$ & $7,6 \pm 0,4$ & $326,4 \pm 39,4$ & $197,1 \pm 9,1$ \\
\hline LV-CA & n.d. & $0,5 \pm 0,2$ & n.d. & $47,4 \pm 15,8$ \\
\hline LV-ER & $14,8 \pm 3,3$ & $5,5 \pm 0,1$ & $463,9 \pm 101,9$ & $170,8 \pm 1,6$ \\
\hline LV-PF & $9,9 \pm 0,1$ & $5,2 \pm 0,5$ & $360,0 \pm 1,8$ & $189,1 \pm 18,2$ \\
\hline LV-SA & $7,3 \pm 0,6$ & $9,5 \pm 0,1$ & $339,5 \pm 30,2$ & $439,5 \pm 2,3$ \\
\hline LB-VA A ${ }_{1}^{\dagger}$ & $17,9 \pm 0,4$ & $6,1 \pm 0,1$ & $477,3 \pm 9,3$ & $162,7 \pm 2,7$ \\
\hline LB-VA A ${ }_{2}^{\ddagger}$ & $5,9 \pm 1,4$ & $12,5 \pm 0,2$ & $220,9 \pm 52,4$ & $470 \pm 5,6$ \\
\hline
\end{tabular}

aLatossolo Vermelho dos municípios de André da Rocha (LV-AR), Cruz Alta (LV-CA), Erechim (LV-ER), Passo Fundo (LV-PF) e Santo Ângelo (LV-SA) e Latossolo Bruno do município de Vacaria (LB-VA). 'Horizonte $\mathrm{A}_{1}$; "horizonte $\mathrm{A}_{2}$. n.d.: não determinado.

Os coeficientes de sorção de mesotriona (Tabela 3) foram mais elevados que os observados por Dyson et al..$^{21} \mathrm{e}$ Chaabane et al..${ }^{22}$ os quais obtiveram valores de $\mathrm{K}_{\mathrm{d}}$ entre 0,13 e $5,0 \mathrm{~L} \mathrm{~kg}^{-1}$ para solos da Europa e EUA. Possivelmente, isso se deve aos baixos teores de argila (6 a $39 \%$ ) e de carbono $(0,6$ a $2,1 \%$ ) dos solos avaliados por aqueles autores, além das diferentes condições experimentais utilizadas. $\mathrm{O}$ $\mathrm{K}_{\mathrm{d}}$ variou de $0,5 \mathrm{~L} \mathrm{~kg}^{-1}$ para o LV-CA a $12,5 \mathrm{~L} \mathrm{~kg}^{-1}$ para o LB-VA A, os quais apresentaram, respectivamente, o menor e o maior teor de argila entre os solos avaliados neste estudo (Tabela 2) indicando que, provavelmente, a fração mineral é o principal sorbente na sorção da mesotriona. A correlação obtida entre o teor de argila e o $\mathrm{K}_{\mathrm{d}}$ corrobora essa hipótese $(r=0,92 ; p<0,01)$. A correlação entre $o K_{d}$ e o teor de carbono das amostras apresentou baixa significância $(r=0,40 ; p<$ 0,38 ), sugerindo que a matéria orgânica possui papel secundário na sorção da mesotriona. Para Dyson et al. ${ }^{21}$ a sorção de mesotriona foi influenciada principalmente pelo $\mathrm{pH}$ do solo e, em menor extensão, pelo teor carbono, sendo favorecida nos solos com os menores valores de $\mathrm{pH}$ e maiores teores de carbono. A contribuição dos constituintes minerais na sorção de mesotriona ainda não foi relatada, embora seja significativa para alguns pesticidas tais como a sulcotriona, um herbicida de caráter ácido fraco com estrutura molecular similar à mesotriona. ${ }^{27}$ Inversamente ao observado para a atrazina, a correlação entre $\mathrm{K}_{\mathrm{d}}$ da mesotriona e o índice C-alquil/C-carboxílico resultou negativa $(\mathrm{r}=-0,69 ; \mathrm{p}<0,08)$, indicando que o aumento da funcionalização da cadeia alifática estimula a sorção do herbicida. Esse resultado, associado à correlação com o teor de argila dos solos estudados, sugere que a sorção da mesotriona envolve uma interação de cargas. Uma vez que os coloides orgânicos e inorgânicos nas condições de $\mathrm{pH}$ das isotermas ( 5,5) apresentam carga de superfície predominantemente aniônica, essa interação ocorre provavelmente via ponte de cátion. Contudo, estudos mais aprofundados serão necessários para comprovar essa hipótese. A ocorrência de interação entre herbicidas ácidos e o solo via ponte de cátion foi sugerida por Chaabane et al. ${ }^{27}$ ao estudarem o processo de retenção de sulcotriona, em quatro solos europeus. Os autores propuseram que o íon $\mathrm{Ca}^{+2}$ atuava como ponte entre o pesticida e os coloides do solo.

\section{Avaliação do potencial de contaminação}

De acordo com os critérios da USEPA, a atrazina e a mesotriona foram classificadas como potenciais contaminantes de águas subterrâneas em todos os solos avaliados. No caso da atrazina apenas os parâmetros especiação (o herbicida encontra-se na sua forma molecular na faixa de $\mathrm{pH}$ dos solos estudados) e $\mathrm{t}_{1 / 2}$ na água não atendem aos critérios de classificação do composto como contaminante em potencial. Para a mesotriona todos os critérios propostos pela USEPA para classificar o composto como contaminante foram atendidos.

Segundo o método GUS, em todos os solos avaliados a atrazina apresentou índice entre 1,8 e 2,8, ou seja, potencial intermediário de lixiviação, indicando a possibilidade de ocorrência de contaminação das águas subterrâneas pelo herbicida. A mesotriona também foi enquadrada na faixa intermediária de lixiviação para a maioria dos solos estudados, exceto no LV-SA e no LB-VA $\mathrm{A}_{2}$, onde o herbicida foi considerado não lixiviável em função do seu elevado $K_{o c}$ nesses solos (Tabela 3). Entretanto, conforme mencionado anteriormente, a matéria orgânica não é o principal sorbente da mesotriona e, portanto, o índice GUS não seria adequado para a avaliação de risco de contaminação por esse herbicida. Adicionalmente, o GUS não considera o transporte de moléculas do pesticida sorvidas às partículas do solo, o que poderia subestimar o potencial contaminante dos produtos. Cabe salientar ainda que o cálculo do GUS é dependente do $t_{1 / 2}$ do pesticida no solo, o qual por sua vez varia com o tipo e uso do solo. ${ }^{20}$ Conforme reportado por Martinazzo et al.,${ }^{20} \mathrm{ot}_{1 / 2}$ da atrazina pode variar de 2,8 a 44,6 dias para um mesmo solo, dependendo do histórico de aplicação do herbicida. Consequentemente, a atrazina poderia ser considerada não lixiviável ou potencialmente lixiviável nesse solo, dependendo do $\mathrm{t}_{1 / 2}$ considerado. Isso enfatiza a importância de se considerar as condições de solo e clima específicas do ambiente de estudo, juntamente com o histórico de aplicação de pesticidas, nas avaliações de risco de contaminação segundo esse método. Muitas vezes a utilização de valores de $t_{1 / 2}$ não representativos da realidade do local de avaliação pode induzir a erros de interpretações quanto ao potencial poluente de um dado pesticida. Exemplo disso são as divergências encontradas nos dados de literatura nas avaliações do potencial de lixiviação de um dado pesticida segundo esse método. 2,21,27,28

Segundo o índice de Goss, a atrazina foi classificada como herbicida de elevado potencial de contaminação de águas superficiais na sua forma dissolvida, enquanto que o potencial de transporte associado 
Tabela 4. Valores dos parâmetros usados no cálculo do ERI para atrazina e mesotriona nos latossolos avaliados

\begin{tabular}{lccccc}
\hline Composto & $\mathrm{t}_{1 / 2}$ dias & Lixiviação LIX & ${\text { Volatilidade } \mathrm{mm} \mathrm{Hg}^{*}}^{\dagger}$ & Perfil toxicológico $^{+}$ & Dose kg ha $^{-1}$ \\
Atrazina & 45,9 & 0,016 & $2,9 \times 10^{-7}$ & 13 & 3,0 \\
Mesotriona & 34,0 & 0,037 & $4,3 \times 10^{-8}$ & 8 & 0,17 \\
\hline
\end{tabular}

$\mathrm{t}_{1 / 2}$ : tempo de meia-vida do composto no solo, ${ }^{*}$ valor médio para os solos avaliados, ${ }^{\dagger}$ valores obtidos pela Equação 4 , ${ }^{+}$valores obtidos pela Equação 5 .

às partículas do solo foi considerado intermediário. Já, a mesotriona foi enquadrada na categoria intermediária de contaminação na forma líquida com baixo potencial de transporte associada às partículas. Resultados similares foram obtidos em estudos anteriores. ${ }^{28}$

O índice de impacto ambiental (Tabela 4) da atrazina, para os solos e condições avaliadas, foi maior que o da mesotriona (ERI = 24 e 6 , respectivamente), indicando que a atrazina possui um risco ambiental estimado quatro vezes maior que a mesotriona. Resultados similares foram reportados por Alister e Kogan. ${ }^{7}$ Tanto a atrazina como a mesotriona são utilizadas em lavouras de produção de milho e possuem espectro de controle de invasoras relativamente semelhante. Sendo assim, para os solos e condições consideradas neste estudo, o uso da mesotriona em detrimento da atrazina seria recomendável. Caso o uso da atrazina se faça necessário, uma alternativa seria a redução da sua dose de aplicação, pois esse foi o fator de impacto que apresentou maior efeito no cálculo do ERI. A simples diminuição da dose de aplicação da atrazina poderia diminuir consideravelmente os riscos de contaminação ambiental. Isso já tem sido feito pelos produtores com o uso de misturas de tanque de atrazina com outros princípios ativos, possibilitando a redução da quantidade aplicada de ambos os produtos e, em geral, o aumento da eficiência de controle das plantas invasoras.

\section{CONCLUSÕES}

A sorção da atrazina foi favorecida nos solos cujo teor e hidrofobicidade da matéria orgânica foram mais elevados. No caso da mesotriona o principal sorbente foi a fração argila e, em menor extensão, a matéria orgânica. Contrariamente ao observado para a atrazina, a sorção desse herbicida foi favorecida pela funcionalização das estruturas alifáticas na composição da matéria orgânica.

A classificação dos herbicidas quanto ao seu potencial de contaminação, de acordo com os critérios teóricos utilizados neste estudo, demonstrou que a atrazina possui um risco potencial elevado de contaminação de águas superficiais e subterrâneas para todos os solos avaliados. Por sua vez, a mesotriona apresenta elevado potencial de risco de contaminação de águas subterrâneas e risco potencial intermediário de contaminação de águas superficiais. Comparativamente à mesotriona, a atrazina apresenta maior índice de impacto ambiental.

Cabe salientar que a utilização de índices e critérios pré-estabelecidos para a avaliação de risco de contaminação por pesticidas conforme realizado neste estudo pode servir de subsídio aos pesquisadores e órgãos ambientais na obtenção de informações preliminares de toxicidade e classificação dos produtos quanto às prioridades de estudo. No entanto, essas informações deverão ser posteriormente associadas aos dados de experimentos e de programas de monitoramento, pois variações de comportamento decorrentes das condições específicas de solo e clima do local de avaliação são esperadas. Considerando-se os diversos parâmetros utilizados nas avaliações de potencial de contaminação, o tempo de meia-vida do composto e os coeficientes de sorção $K_{d}$ e $K_{o c}$ merecem especial atenção dos modeladores por apresentarem grande variabilidade entre solos. Entre os diferentes métodos de avaliação disponíveis na literatura, deve-se dar preferência aos que fazem uso de ampla variedade de parâmetros para melhor diferenciação dos pesticidas.

\section{MATERIAL SUPLEMENTAR}

As Figuras 1S (Estrutura molecular da atrazina e da mesotriona) e $2 \mathrm{~S}$ (Isotermas de sorção de mesotriona em amostras de horizonte A de Latossolo Vermelho dos municípios de André da Rocha (LVAR), Cruz Alta (LV-CA), Erechim (LV-ER), Passo Fundo (LV-PF) e Santo Ângelo (LV-SA), e em amostras de horizonte $\mathrm{A}_{1}$ e $\mathrm{A}_{2}$ de Latossolo Bruno do município de Vacaria (LB-VA)) e as Tabelas $1 \mathrm{~S}$ (Coeficientes de sorção $\left(\mathrm{K}_{\mathrm{d}}\right)$ de atrazina em solos brasileiros) e $2 \mathrm{~S}$ (Distribuição dos grupos funcionais de carbono determinados por espectroscopia de ${ }^{13} \mathrm{C}$ RMN CP/MAS e índice C-alquil/C-carboxílico em amostras de matéria orgânica dos latossolos avaliados) encontramse disponíveis gratuitamente em http://quimicanova.sbq.org.br, na forma de arquivo PDF.

\section{AGRADECIMENTOS}

Ao Conselho Nacional de Desenvolvimento Científico e Tecnológico $(\mathrm{CNPq})$ pela concessão das bolsas de produtividade e de doutorado e ao convênio $\mathrm{CNPq} / \mathrm{DAAD}$ pelo apoio financeiro à pesquisa.

\section{REFERÊNCIAS}

1. Graymore, M.; Stagnitti, F.; Allinson, G.; Environ. Int. 2001, 26, 483.

2. Dores, E. F. G. C.; De-Lamonica-Freire, E. M.; Quim. Nova 2001, 24, 27.

3. Silva, D. R. O.; Avila, L. A.; Agostinetto, D.; Dal Magro, T.; Oliveira, E.; Zanella, R.; Noldin, J. A.; Cienc. Rural, 2009, 39, 2383.

4. Cohen, S. Z.; Wauchope, R. D.; Klein, A. W. E.; Eadsforth, C. V.; Graney, R.; Pure Appl .Chem. 1995, 67, 2109.

5. Gustafson, D. I.; Environ. Toxicol. Chem. 1989, 8, 339.

6. Goss, D. W.; Weed Technol. 1992, 6, 701.

7. Alister, C.; Kogan, M.; Crop Prot. 2006, 25, 202.

8. Primel, E. G.; Zanella, R.; Kurz, M. H. S.; Gonçalves, F. F.; Machado, S. O.; Marchezan, E.; Quim. Nova 2005, 28, 605.

9. Pessoa, M. C. P. Y.; Scramin, S. Em Modelagem matemática e simulação da exposição do ambiente à aplicação de agrotóxicos: apoio à avaliação de risco ambiental; Silva, C. M. S.; Fay, E. F., eds.; Embrapa Informação Tecnológica: Brasília, 2004, cap. 9.

10. Armas, E. D.; Monteiro, R. T. R.; Amâncio, A. V.; Correa, R. M. L.; Guercio, M. A.; Quim. Nova 2005, 28, 975.

11. Armel, G. R.; Wilson, H. P.; Richardson, R. J.; Hines, T. E.; Weed Technol. 2003, 17, 284; Armel, G. R.; Hall, G. J.; Wilson, H. P.; Cullen, N.; Weed Sci. 2005, 53, 202; Abendroth, J. A.; Martin, A. R.; Roeth, F. W.; Weed Technol. 2006, 20, 267; Creech, J. E.; Monaco, T. A.; Evans, J. O.; Pest Manage. Sci. 2004, 60, 1079.

12. Tedesco, M. J.; Gianello, C.; Bissani, C. A.; Bohnen, H.; Volkweiss, S. J.; Análise de solo, plantas e outros materiais, $2^{\mathrm{a}}$ ed., Universidade Federal do Rio Grande do Sul: Porto Alegre, 1995.

13. Dick, D. P.; Gonçalves, C. N.; Dalmolin, R. S. D.; Knicker, H.; Klamt, E.; Kögel-Knabner, I. ; Simões, M. L.; Martin-Neto, L.; Geoderma 2005, 124, 319.

14. Dalmolin, R. S. D.; Gonçalves, C. N.; Dick, D. P.; Knicker, H.; Klamt, E.; Kögel-Knabner, I.; Eur. J. Soil Sci. 2006, 57, 644.

15. http://www.eu-footprint.org, acessada em Maio 2011.

16. Ávila, L. G.; Leite, S. B.; Dick, D. P.; Pohlmann, A.; Quim. Nova 2009, 32, 1727. 
17. Kleinschmitt, A. R. B.; Tese de Doutorado, Universidade Federal do Rio Grande do Sul, Brasil, 2007.

18. Hirsch, M. M.; Monografia, Universidade Federal do Rio Grande do Sul, Brasil, 2008.

19. Souza, M. D.; Boeira, R. C.; Gomes, M. A. F.; Ferracini, V. L.; Maia, A. H. N.; Rev. Bras. Cienc. Solo 2001, 25, 1053.

20. Martinazzo, R.; Jablonowski, N. D.; Hamacher, G.; Dick, D. P.; Burauel, P.; J. Agric. Food Chem. 2010, 58, 7864.

21. Dyson, J. S.; Beulke, S.; Brown, C. D.; Lane, M. C. G.; J. Environ. Qual. 2002, 31, 613.

22. Chaabane, H.; Vulliet, E.; Calvayrac, C.; Coste, C. M.; Cooper, J. F.; Pest Manage. Sci. 2008, 64, 86.

23. Spadotto, A. C.; Pesticidas: Rev. Ecotoxicol. Meio Amb. 2002, 12, 69.
24. Oliveira Jr., R. S.; Koskinen, W. C.; Ferreira, F. A.; Weed Res. 2001, 41, 97.

25. Dick, D. P.; Martinazzo, R.; Knicker, H.; Almeida, P. S. G.; Quim. Nova 2010, 33, 14 .

26. Piccolo, A.; Conte, P.; Scheunert, I.; Paci, M.; J. Environ. Qual. 1998, 27, 1324.

27. Chaabane, H.; Cooper, J. F.; Azouzi, L.; Coste, C. M.; J. Agric. Food Chem. 2005, 53, 4091.

28. Milhome, M. A. L.; Sousa, D. O. B.; Lima, F. A. F.; Nascimento, R. F.; Eng. Sanit. Ambient. 2009, 14, 363.

29. Santana, G. S.; Dissertação de Mestrado, Universidade Federal do Rio Grande do Sul, Brasil, 2010. 


\section{Rosane Martinazzo*}

Departamento de Solos, Faculdade de Agronomia, Universidade Federal do Rio Grande do Sul, Av. Bento Gonçalves, 7712 , 91501-970 Porto Alegre - RS, Brasil

Deborah Pinheiro Dick, Mauro Mozael Hirsch e Simone Benvenuti Leite

Departamento de Físico-Química, Instituto de Química, Universidade Federal do Rio Grande do Sul, Av. Bento Gonçalves, 9500 , 91501-970 Porto Alegre - RS, Brasil

Maria do Carmo Ruaro Peralba

Departamento de Química Inorgânica, Instituto de Química, Universidade Federal do Rio Grande do Sul, Av. Bento Gonçalves, 9500, 91501-970 Porto Alegre - RS, Brasil

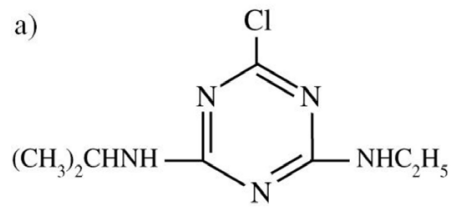

b)<smiles>CC1(C)CCCC(=O)C1(C)C(=O)c1ccc(S(C)(=O)=O)cc1[N+](=O)[O-]</smiles>

Figura 1S. Estrutura molecular da atrazina (a) e da mesotriona (b). Fontes: refs. 1 e 2

Tabela 1S. Classificação, local e profundidade de amostragem, teores de argila e de carbono e coeficientes de sorção ( $\left.\mathrm{K}_{\mathrm{d}}\right)$ de atrazina de amostras de solos brasileiros

\begin{tabular}{|c|c|c|c|c|c|c|}
\hline Solo & Local & $\begin{array}{l}\text { Prof. } \\
(\mathrm{cm})\end{array}$ & $\begin{array}{c}\text { Argila } \\
(\%)\end{array}$ & $\begin{array}{c}\mathrm{C} \\
(\%) \\
\end{array}$ & $\begin{array}{c}\mathrm{K}_{\mathrm{d}} \\
\left(\mathrm{L} \mathrm{kg}^{-1}\right) \\
\end{array}$ & Ref. \\
\hline Argissolo Vermelho Amarelo & Seropédica - RJ & $0-10$ & 24,0 & 2,80 & 1,28 & 3 \\
\hline Latossolo Roxo & Capinópolis - MG & $0-20$ & 42,0 & 1,45 & 4,06 & 4 \\
\hline Latossolo Roxo & Londrina - PR & $0-20$ & 75,0 & 2,78 & 1,69 & 4 \\
\hline Areia Quartzoza & Mocambinho - MG & $0-20$ & 10,0 & 0,35 & 0,51 & 4 \\
\hline Areia Quartzoza & Venda Nova - ES & $0-20$ & 14,0 & 7,45 & 10,5 & 4 \\
\hline Argissolo Vermelho & Eldorado do Sul - RS & $0-10$ & 21,3 & 1,45 & 3,6 & 5 \\
\hline Vertissolo Ebânico & Aceguá - RS & $0-10$ & 38,0 & 3,31 & 11,5 & 5 \\
\hline Latossolo Vermelho & Ijuí - RS & $0-10$ & 59,6 & 2,30 & 7,4 & 5 \\
\hline Planossolo Háplico & Pelotas - RS & $0-10$ & 85,0 & 1,22 & 4,3 & 5 \\
\hline Latossolo Vermelho & Ibirubá - RS & $0-1$ & 32,0 & 2,38 & 22,0 & 6 \\
\hline Latossolo Vermelho & Ibirubá - RS & $5-20$ & 30,0 & 1,51 & 25,0 & 6 \\
\hline Latossolo Vermelho & Ponta Grossa - PR & $0-20$ & 47,0 & 2,90 & 3,88 & 7 \\
\hline Latossolo Vermelho Amarelo & Sub-bacia do Rio das Mortes - MG & $0-20$ & 53,0 & 2,40 & 5,96 & 8 \\
\hline Cambissolo Háplico & Sub-bacia do Rio das Mortes - MG & $0-20$ & 58,3 & 2,64 & 2,16 & 8 \\
\hline Neossolo Flúvico & Sub-bacia do Rio das Mortes - MG & $0-20$ & 23,3 & 2,00 & 1,34 & 8 \\
\hline Latossolo Amarelo & Sub-bacia do Rio das Mortes - MG & $0-20$ & 32,7 & 2,72 & 2,86 & 8 \\
\hline Latossolo Vermelho & Sub-bacia do Rio das Mortes - MG & $0-20$ & 34,0 & 2,16 & 4,87 & 8 \\
\hline Glei Húmico & Indaiatuba $-\mathrm{SP}$ & n.d. & 27,9 & 2,09 & 2,6 & 9 \\
\hline Latossolo Vermelho escuro & Campinas - SP & n.d. & 44,9 & 2,09 & 2,1 & 9 \\
\hline Latossolo Bruno & Vacaria - RS & $0-30$ & 52,4 & 3,51 & 12,0 & 10 \\
\hline Latossolo Vermelho & Augusto Pestana - RS & $0-23$ & 62,5 & 2,25 & 14,2 & 10 \\
\hline Latossolo Vermelho Amarelo & Brasília - DF & $0-19$ & 63,3 & 2,62 & 14,6 & 10 \\
\hline
\end{tabular}

n.d.: não disponível

*e-mail: martinazzo_ro@yahoo.com.br 

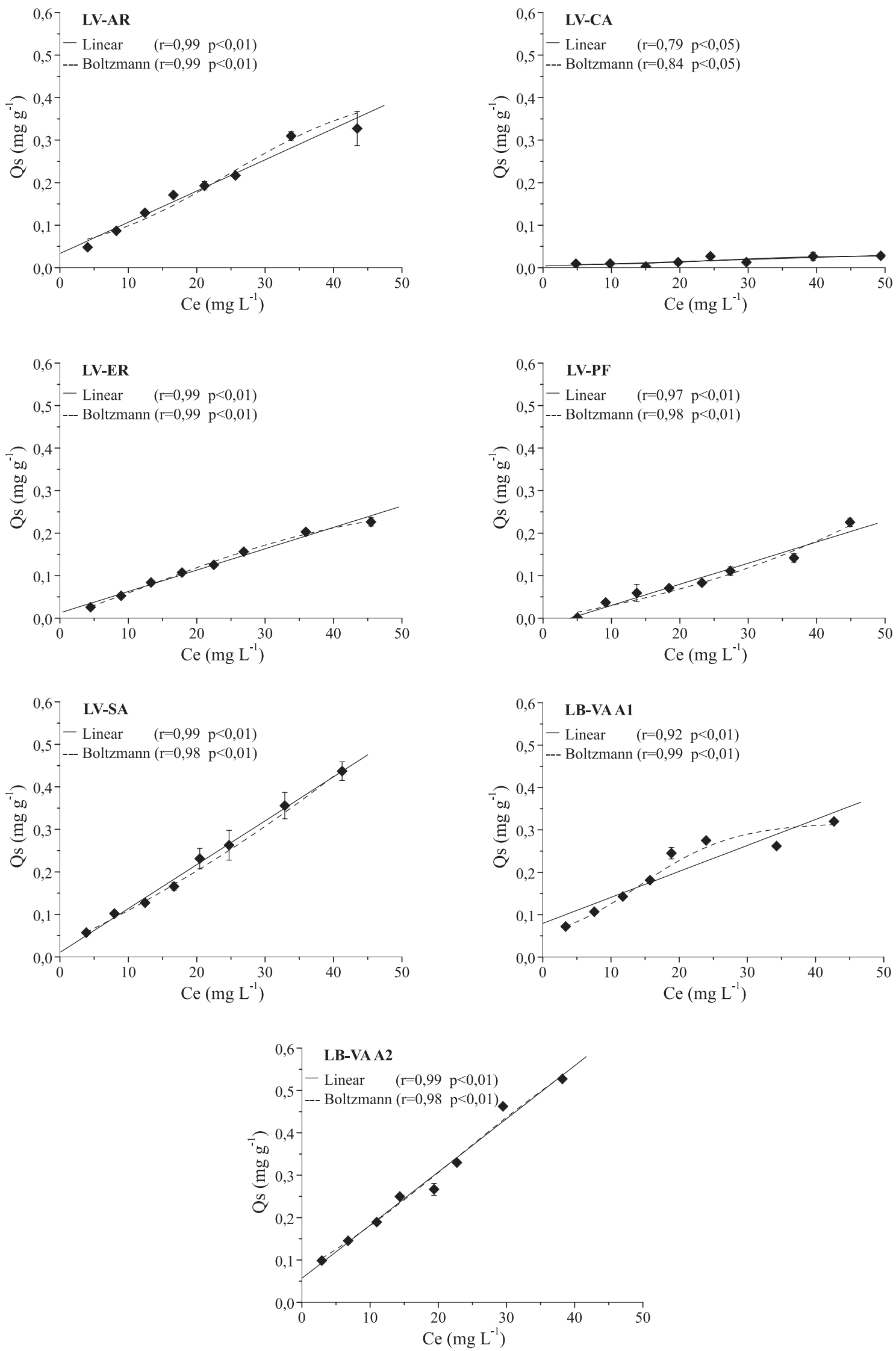

Figura 2S. Isotermas de sorção de mesotriona em amostras de horizonte A de Latossolo Vermelho dos municípios de André da Rocha (LV-AR), Cruz Alta (LV$C A)$, Erechim (LV-ER), Passo Fundo (LV-PF) e Santo Ângelo (LV-SA), e em amostras de horizonte $A_{1}$ e $A_{2}$ de Latossolo Bruno do município de Vacaria (LB-VA). Os símbolos do gráfico representam a média de duas repetições e as barras de erro indicam o desvio padrão da média e não aparecem quando menores que o símbolo para a média 
Tabela 2S. Distribuição dos grupos funcionais de carbono determinados por espectroscopia de ${ }^{13} \mathrm{C}$ RMN CP/MAS e índice C-alquil/C-carboxílico em amostras de matéria orgânica dos latossolos avaliados

\begin{tabular}{|c|c|c|c|c|c|}
\hline Solo $^{\mathrm{a}}$ & Alquil (0-45) & \multicolumn{2}{|c|}{ Tipo de C/deslocamento químico (ppm) } & \multirow{2}{*}{ Carboxílico (160-230) } & \multirow{2}{*}{ C-alquil/ C-carboxílico } \\
\hline & & \multicolumn{2}{|c|}{$(\%)$} & & \\
\hline LV-CA & 23,5 & 43,7 & 20,6 & 12,4 & 1,90 \\
\hline LV-ER & 24,4 & 45,3 & 17,6 & 12,6 & 1,94 \\
\hline LV-SA & 24,0 & 45,9 & 15,9 & 14,2 & 1,69 \\
\hline LB-VA $A_{1}$ & 25,0 & 48,0 & 14,0 & 13,0 & 1,92 \\
\hline LB-VA A 2 & 23,8 & 41,1 & 20,1 & 15,0 & 1,59 \\
\hline
\end{tabular}

${ }^{2}$ Latossolo Vermelho dos municípios de André da Rocha (LV-AR), Cruz Alta (LV-CA), Erechim (LV-ER), Passo Fundo (LV-PF), Santo Ângelo (LV-SA) e Latossolo Bruno do município de Vacaria (LB-VA). Fontes: refs. 11 e 12

\section{REFERÊNCIAS}

1. http://farmchemicalsinternational.com/cropprotection/cpd, acessada em Maio 2011.

2. Dyson, J. S.; Beulke, S.; Brown, C. D.; Lane, M. C. G.; J. Environ. Qual. 2002, 31, 613

3. Correia, F.; Mercante, F.; Fabrício, A.; Campos, T.; Vargas Jr., E.; Langenbach, T.; Pesticidas: Rev. Ecotoxicol. Meio Amb. 2007, 17, 37.

4. Oliveira Jr., R. S.; Koskinen, W. C.; Ferreira, F. A.; Weed Res. 2001, 41, 97.

5. Dick, D. P.; Martinazzo, R.; Knicker, H.; Almeida, P. S. G.; Quim. Nova 2010, 33, 14 .

6. Kleinschmitt, A. R. B.; Tese de Doutorado, Universidade Federal do Rio Grande do Sul, Brasil, 2007.

7. Prata, F.; Lavorenti, A.; Vanderborght, J.; Burauel, P.; Vereecken, H.; Vadose Zone J. 2003, 2, 728.
8. Arantes, S. A. C. M.; Lima, J. M.; Nóbrega, J. C. A.; Guilherme, 1. R. G.; Julião, 1. G. F.; Jesus, E. A.; Pesticidas: Rev. Ecotoxicol. Meio Amb. 2006, 16, 101 .

9. Nakagawa, L. E.; Luchini, L. C.; Musumeci, M. R.; Andréa, M. M.; Pesqui. Agropecu. Bras. 1995, 30, 471.

10. Ávila, L. G.; Leite, S. B.; Dick, D. P.; Pohlmann, A.; Quim. Nova 2009, 32, 1727.

11. Dick, D. P.; Gonçalves, C. N.; Dalmolin, R. S. D.; Knicker, H.; Klamt, E.; Kögel-Knabner, I. ; Simões, M. L.; Martin-Neto, L.; Geoderma 2005, 124, 319.

12. Dalmolin, R. S. D.; Gonçalves, C. N.; Dick, D. P.; Knicker, H.; Klamt, E.; Kögel-Knabner, I.; Eur. J. Soil Sci. 2006, 57, 644. 